ARTÍ́CULO

\title{
Presencia de atún lanzón en la costa sur de Chile: Potencial nuevo recurso para la pesca artesanal
}

Presence of slender tuna in the south coast of Chile: Potential new resource for artisanal fishing

Pablo R. Reyes ${ }^{1 *}$, Mathias Hüne ${ }^{1}$, Francisco Olguín ${ }^{2}$ y Víctor Moncada ${ }^{1}$

${ }^{1}$ Fundación Ictiológica, Willie Arthur 2030/704, Providencia, Santiago, Chile

${ }^{2}$ Instituto de Ciencias Marinas y Limnológicas, Universidad Austral de Chile, Casilla 5090000, Valdivia, Chile

*Autor corresponsal: preyes@ictiologica.org

\begin{abstract}
Current investigation analysed an abundant and massive presence of "slender tuna" Allothunnus fallai through 1,026 nautical miles in the southern Chile coastline ( $37^{\circ} 03^{\prime}-51^{\circ} 49^{\prime}$ S), between 2014 and 2017, included its presence inner the Magellanic channels of Chilean Patagonia. We refuse that the presence of the species is linked with coastal SST increases derived from El Niño, which arrival to the Chilean coast would be associated to lowered primary oceanic production, causing an approach to Chilean coastal waters in search of food. Due its abundance in 2017 and 2018 Chilean State has formalized the "slender tuna" as a new Chilean official artisan fishing resource.
\end{abstract}

Key words: Allothunnus fallai, Black Tuna, Southeaster Pacific, Patagonia

Resumen.- La presente investigación analizó la abundante y masiva presencia de "atún lanzón", Allothunnus fallai en 1.026 m.n. costeras del sur de Chile ( $37^{\circ} 03^{\prime}-51^{\circ} 49^{\prime}$ S), entre 2014 y 2017, incluidos el interior de los canales Magallánicos de Patagonia chilena. Se descartó que incrementos locales de la TSM provocados por El Niño causaran la presencia de la especie, cuyo arribo a la costa chilena se asociaría a la disminución de la producción primaria oceánica, provocando un acercamiento a la costa en busca de alimento. Producto de su abundancia, en 2017 y 2018 el Estado Chileno oficializó al “atún lanzón” como nuevo recurso pesquero artesanal chileno.

Palabras clave: Allothunnus fallai, atún negro, Pacífico Suroriental, Patagonia

\section{INTRODUCCIÓN}

A lo largo de la costa de Chile se ha reportado la presencia ocasional de una veintena de especies de la Familia Scombridae, destacando en las estadísticas pesqueras Gasterochisma melampus Richardson, 1845; Thunnus alalunga (Bonnaterre, 1788); Katsuwonus pelamis (Linnaeus, 1758); Scomber japonicus Houttuyn, 1782, T. obesus (Lowe, 1839); T. albacares Bonnaterre, 1788 y T. maccoyii (Castelnau, 1872) (Fowler 1945, Bahamonde \& Pequeño 1975, Pequeño 1989, 1997, Arana et al. 2012, Reyes \& Hüne 2012, SERNAPESCA 2012, 2016).

Una de las más escasas especies de atún en la costa chilena es Allothunnus fallai (Serventy, 1948), registrada por primera y única vez en el Fiordo Aysén (45 $15^{\prime}$ 'S-73 $\left.{ }^{\circ} 15^{\prime} \mathrm{O}\right)$ durante El Niño 1982-1983 (Zama \& Cardenas 1984). Dado que estos eventos generalmente no afectan latitudes mayores a $40^{\circ} \mathrm{S}$ (e.g., El Niño 1997 alcanzó los 40S) (Carr et al. 2002), El Niño 82-83 fue calificado como "Meganiño 1982-83" (Rocha 2007) y "Particularmente Intenso" (Maturana et al. 1997). A. fallai, sin embargo, ha sido registrada hasta los $53^{\circ} 25^{\prime} \mathrm{S}$ en la Patagonia atlántica (Collette \& Díaz de Astarloa 2008), lo que se consideró posible gracias a estrategias fisiológicas que le permiten incrementar las temperaturas de los músculos rojos y su temperatura cerebral hasta $9{ }^{\circ} \mathrm{C}$ por sobre la temperatura ambiental, conservando el calor metabólico generado por la natación continua (Graham \& Dickson 2000, Sepúlveda et al. 2007, 2008). El hábitat de la especie se encuentra entre los paralelos $20^{\circ} \mathrm{S}$ y $54^{\circ} \mathrm{S}$ de los océanos Índico, Atlántico y Pacífico (Collette \& Nauen 1983, Eschmeyer et al. 1983, Collette \& Díaz de Astarloa 2008, Pepperell \& Harvey 2010). Pese a su gran distribución geográfica, en aguas costeras de Chile sólo existe un registro de la especie, lo que explica la ausencia de información científica biológica o pesquera, tampoco registros oficiales de desembarques, ni especímenes en colecciones zoológicas (Bahamonde \& Pequeño 1975, Pequeño 1989, Meléndez et al. 1993, SERNAPESCA 2012, 2016).

Fuera de la Zona Económica Exclusiva (ZEE) de Chile, en el Pacífico Sur $\left(150-80^{\circ} \mathrm{O}\right.$ área FAO 81) existe una pesquería que entre 1999-2011 presentó capturas promedio anuales de 51 ton (FAO 2018), concentrándose las capturas durante noviembre y diciembre cerca de la ZEE chilena (Warashina \& Hisada 1972, Ichikawa \& Shirasawa 1980, Collette \& Nauen 1983, Yatsu 1995). Según Yatsu (1995) su rol ecológico en el ecosistema pelágico del Pacífico Suroriental la sitúa como especie dominante: los juveniles se encuentran principalmente entre los 20 y $35^{\circ} \mathrm{S}$ a una Temperatura Superficial del Mar (TSM) que varía de 19 a $24{ }^{\circ} \mathrm{C}$. Durante primavera austral (octubre, noviembre 
y diciembre) se desplazan a desovar al norte de los $31^{\circ} \mathrm{S}$, en aguas subtropicales con TSM de $17-25^{\circ} \mathrm{C}$ (Watanabe et al. 1966, Yatsu 1995), luego en primavera y verano austral (noviembre a febrero) migran al sur de los $38^{\circ} \mathrm{S}$ para alimentarse de plancton hasta el fin del verano austral o comienzos del otoño austral (marzo), cuando regresan a aguas subtropicales a reproducirse. Dicha migración estacional cubre más de 4.000 millas náuticas (mn) y constituye un importante eslabón ecológico al transportar energía desde los productivos ecosistemas pelágicos subantárticos a los subtropicales. Los individuos de mayor tamaño gradualmente alcanzan mayores latitudes, con menores temperaturas (Mori 1967, Collette \& Nauen 1983, Yatsu 1995). Si bien la especie no ingresa en la ZEE de Chile, si proporciona energía a grandes peces pelágicos que constituyen recursos pesqueros para Chile [e.g., Isurus oxyrinchus Rafinesque, 1810; Prionace glauca (Linnaeus, 1758); Xiphias gladius Linnaeus, 1758 y Makaira mazara (Jordan \& Snyder, 1901), entre otros] (Yatsu 1995, SERNAPESCA 2016). A diferencia de la ausencia observada en aguas costeras chilenas, en Nueva Zelanda la especie se acerca para forrajear "krill" (Euphausiacea), junto con Thyrsites atun (Euphrasen, 1791) y Trachurus murphyi Nichols, 1920 (O’Driscoll \& McClatchie 1998).

La histórica y continua escasez de la especie en aguas chilenas, salvo el registro único de 1982 (Zama \& Cárdenas 1984), fue interrumpido desde 2014 hasta el verano de
2019, debido a abundantes y continuas capturas en aguas costeras realizadas por la flota pesquera artesanal y la pesca recreativa. Lo anterior implicó que el Estado oficializara sus capturas entre los $18^{\circ} 28^{\prime} \mathrm{S}$ y $41^{\circ} 28^{\prime}$ 'S (SUBPESCA 2017, 2018), aunque existen capturas registradas hasta los $51^{\circ} 49^{\prime} \mathrm{S}$.

En el presente trabajo se analizaron las circunstancias ambientales que permitieron el acceso de especímenes hasta la costa sur de Chile, entre los $37^{\circ} 03^{\prime}$ y $51^{\circ} 49^{\prime} \mathrm{S}$, bajo la hipótesis que su masiva llegada ocurrió por El Niño 201416, tal como sucedió durante el "Meganiño 82-83" (Zama \& Cárdenas 1982, Strub et al. 1998, Blanco et al. 2002).

\section{MATeriales Y MÉTODOS}

Durante 51 meses (noviembre 2014-febrero 2019) de capturas de la especie en Chile, el presente estudio seleccionó 10 de los registros disponibles de $A$. fallai para su análisis. Todos los registros analizados ocurrieron entre noviembre 2014 y mayo 2017, entre la Isla Santa María (SM) de la región del Biobío (3703’S) y Puerto Riquelme (PR) en la región de Magallanes (5149'S), puntos distanciados por una distancia lineal de $1.026 \mathrm{mn}$ de costa chilena (Fig. 1), con decenas de registros generados por su abundancia, que llevó a la especie a estar disponible en los mercados de peces, entre ellos en el mercado de Angelmó, en Puerto Montt, Los Lagos (El Llanquihue 2017) ${ }^{1}$ y el Mercado Fluvial de Valdivia, Los Ríos (Fig. 2 y registro Bahía de Coral en Tabla 1).
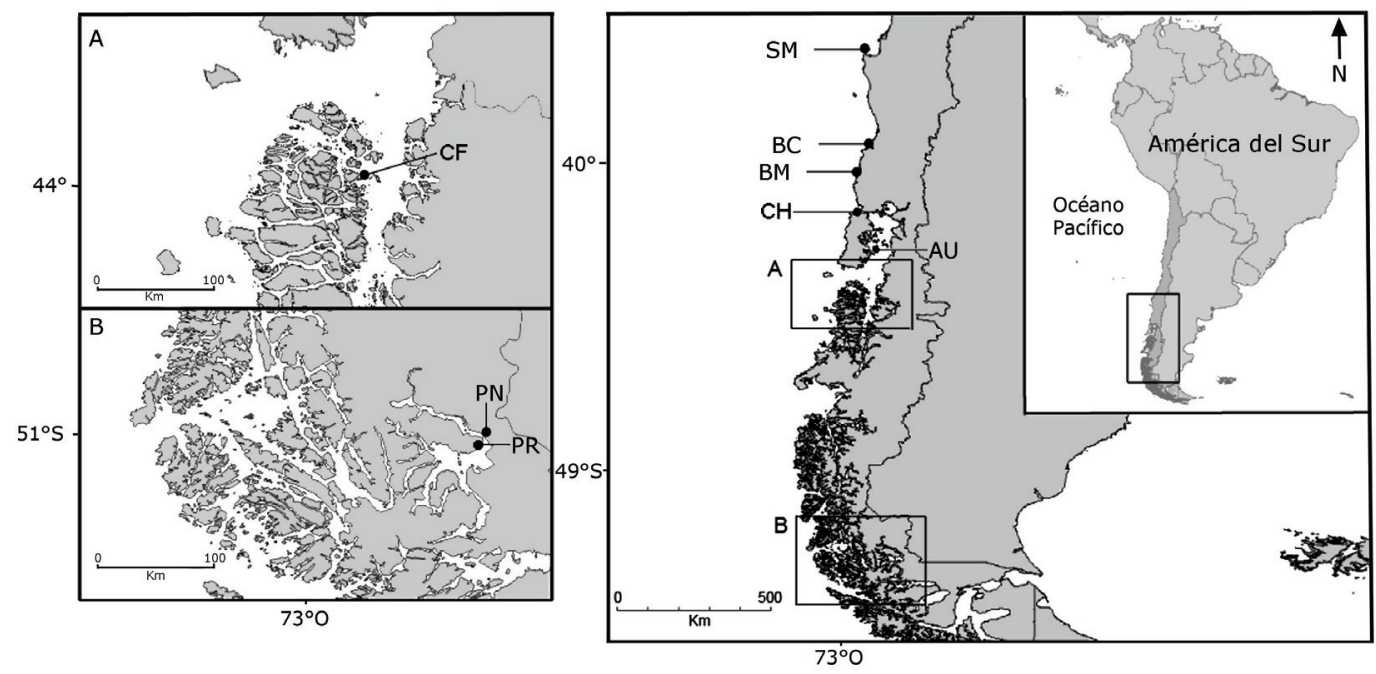

Figura 1. Área de estudio y registro de atunes entre 2014 y 2017. SM (Isla Santa María), CM (Caleta Mehuín), BC (Bahía de Corral), BM (Bahía Mansa), CH (Canal de Chacao), AU (Auchemó), CF (canal Frödden) (Recuadro A), PN (Puerto Natales) y PR (Puerto Riquelme) (Recuadro B) / Study area and tuna records between 2014 and 2017. SM (Santa Maria Island), CM (Mehuín Caleta's), BC (Corral Bay), BM (Mansa Bay), CH (Chacao Channel), AU (Auchemó), CF (Frödden Channel) (Frame A), PN (Natales Port) and PR (Riquelme Port) (Frame B)

\footnotetext{
${ }^{1}$ El Llanquihue. 2017. Atún elongado, el pez que llegó a potenciar la variada cocina local. Tema del Día, pp. 2 y 3. Diario El Llanquihue de Puerto Montt, domingo 14 de mayo 2017. <https://pbs.twimg.com/media/C_zSKO_XgAEtidD.jpg>

${ }^{2}<$ https://www.ncdc.noaa.gov/>
} 
Tabla 1. Antecedentes de los ejemplares estudiados en la zona de esta investigación; nombre del lugar de registro, código del espécimen, coordenadas, fecha, pesquería, TSM (Temperatura Superficial del Mar), $\triangle T S M$ (anomalía de la TSM) e índice oceánico ENOS (ONI), de cada registro considerado. Especímenes BC corresponden a MNHNCL ICT 7536-1 y MNHNCL ICT 7536-2 / Data of the specimens considered on the studied area during this research; name of the registration place, specimen code, coordinates, date, fishery, TSM (Sea Surface Temperature), $\triangle$ TSM (anomaly of the SST) and oceanic index ENSO (ONI) to each considerated record. BC specimens correspond to MNHNCL ICT 7536-1 and MNHNCL ICT 7536-2

\begin{tabular}{|c|c|c|c|c|c|c|c|c|}
\hline Lugar & Código & Latitud & Longitud & $\begin{array}{c}\text { Fecha } \\
\text { (aa,MM,dd) }\end{array}$ & $\begin{array}{l}\text { Captura } \\
\text { asociada }\end{array}$ & $\begin{array}{l}\text { TSM } \\
\left({ }^{\circ} \mathrm{C}\right)\end{array}$ & $\begin{array}{l}\Delta \mathrm{TSM} \\
\left({ }^{\circ} \mathrm{C}\right)\end{array}$ & $\mathrm{ONI}\left({ }^{\circ} \mathrm{C}\right)$ \\
\hline $\begin{array}{l}\text { Pto. Natales, } \\
\text { Magallanes }\end{array}$ & PN & $51^{\circ} 44.2^{\prime} \mathrm{S}$ & $72^{\circ} 30.3^{\prime} \mathrm{O}$ & $14, N O V, 01$ & $\begin{array}{l}\text { Ejemplar varado } \\
\text { en costa }\end{array}$ & 8,4 & 0,1 & $+0,52$ \\
\hline $\begin{array}{l}\text { Bha. Mansa, } \\
\text { Los Lagos }\end{array}$ & $\mathrm{BM}$ & $40^{\circ} 33^{\prime} \mathrm{S}$ & $73^{\circ} 46^{\prime} \mathrm{O}$ & 15, MAY-JUN & T. atun & 11,2 & $-0,3$ & $+0,84 \mathrm{y}+0,97$ \\
\hline $\begin{array}{l}\text { Pto. Riquelme, } \\
\text { Magallanes }\end{array}$ & PR & $51^{\circ} 49^{\prime} \mathrm{S}$ & $72^{\circ} 38^{\prime} \mathrm{O}$ & 16,MAR, 12 & M. australis & 11,7 & $-3,0$ & $+1,58$ \\
\hline $\begin{array}{l}\text { Auchemó, } \\
\text { Los Lagos }\end{array}$ & $\mathrm{AU}$ & $43^{\circ} 01^{\prime} \mathrm{S}$ & $72^{\circ} 49^{\prime} \mathrm{O}$ & $16, \mathrm{MAY}, 15$ & O. tshawytscha & 13,8 & 0,8 & $+0,59$ \\
\hline $\begin{array}{l}\text { Bha. Mansa, } \\
\text { Los Lagos }\end{array}$ & BM II & $40^{\circ} 33^{\prime} \mathrm{S}$ & $73^{\circ} 46^{\prime} \mathrm{O}$ & 16,ENE-ABR & T. atun & 10,3 & 0,8 & $+2,22-+1,08$ \\
\hline $\begin{array}{l}\text { Bha. Corral. } \\
\text { Los Ríos }\end{array}$ & $\mathrm{BC}$ & $39^{\circ} 52^{\prime} \mathrm{S}$ & $73^{\circ} 26^{\prime} \mathrm{O}$ & 16,JUN, 10 & T. atun & 13,0 & 0,3 & $-0,09$ \\
\hline $\begin{array}{l}\text { Mehuín. } \\
\text { Los Ríos }\end{array}$ & $\mathrm{CM}$ & $39^{\circ} 26^{\prime} \mathrm{S}$ & $73^{\circ} 10^{\prime} \mathrm{O}$ & $16, \mathrm{AGO}, 25$ & T. atun & 11,2 & $-0,4$ & $-0,59$ \\
\hline $\begin{array}{l}\text { I. Sta María, } \\
\text { Biobío }\end{array}$ & SM & $37^{\circ} 03^{\prime} \mathrm{S}$ & $73^{\circ} 31^{\prime} \mathrm{O}$ & $17, \mathrm{FEB}, 14-27$ & T. atun & 13,6 & $-1,0$ & $-0,2$ \\
\hline $\begin{array}{l}\text { Canal Frödden, } \\
\text { Aysén }\end{array}$ & $\mathrm{CF}$ & $44^{\circ} 16^{\prime} \mathrm{S}$ & $73^{\circ} 34^{\prime} \mathrm{O}$ & $17, \mathrm{FEB}$ & M. australis & 15,2 & 0,4 & $-0,2$ \\
\hline $\begin{array}{l}\text { Canal de Chacao, } \\
\text { Los Lagos }\end{array}$ & $\mathrm{CH}$ & $41^{\circ} 50^{\prime} \mathrm{S}$ & $73^{\circ} 32^{\prime} \mathrm{O}$ & 17,MAY,06 & O. tshawytscha & 13,8 & 0,6 & $+0,2$ \\
\hline
\end{tabular}

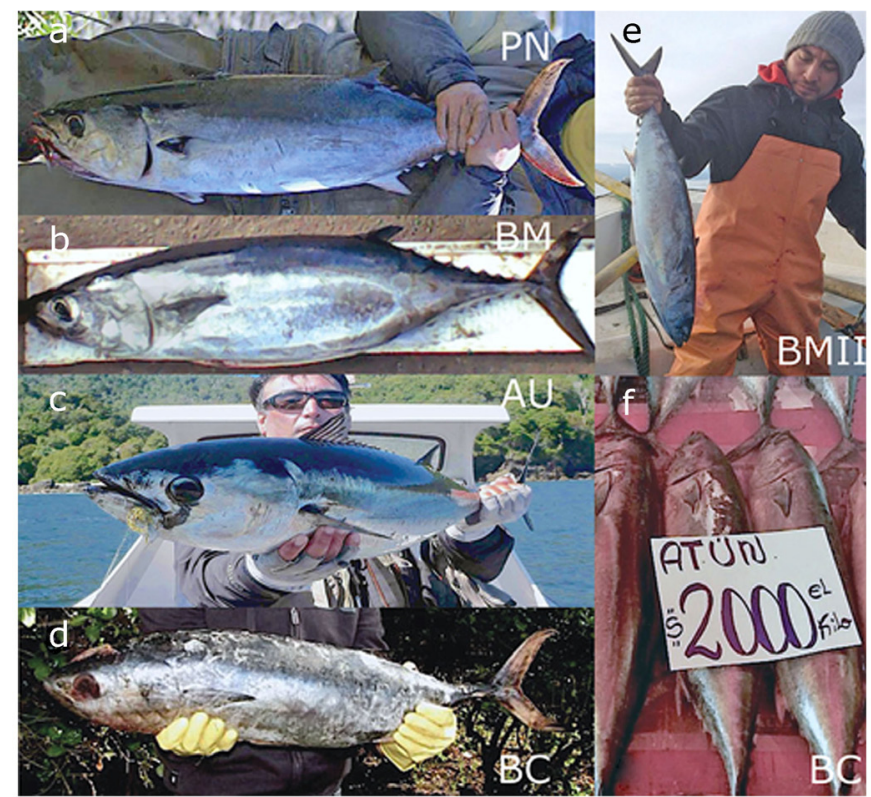

Figura 2. A. fallai estudiados en el sur de Chile: a) PN, Puerto Natales 2014; b) BM, Bahía Mansa 2015; c) AU, Auchemó 2016 (capturado como pesca recreativa); d) BC 2016, corresponde a ejemplar capturado en la Bahía de Corral para su donación al Museo Nacional de Historia Natural de Chile (MNHNCL); e) BM II, Bahía Mansa 2016; f) BC 2016, ejemplares capturados en la Bahía de Corral, a la venta en el Mercado Fluvial de Valdivia, Los Ríos / A. fallai studied on southern Chile: a) PN, Puerto Natales 2014; b) BM, Bahía Mansa 2015; c) AU, Auchemó 2016 (caught as recreational fishing); d) BC 2016, corresponds to a specimen captured in the Corral Bay to be donated to the Chilean National Museum of Natural History (MNHNCL); e) BM II, Bahía Mansa 2016; and f) BC 2016, specimens captured in the Corral Bay, for sale in the Fluvial Market of Valdivia, Los Ríos 
Cada observación fue analizada en función del comportamiento de El Niño Oscilación del Sur (ENOS), con datos satelitales obtenidos del NCEI de la NOAA ${ }^{2}$ e imágenes AVHRR de la región Niño 3.4. También se analizaron en base a los valores de la NOAA para TMS y de Anomalía ( $\triangle \mathrm{TSM}$ ) (Tablas 1 y 2 ). Se considera evento de El Niño cuando el valor promedio en tres meses de su índice Oceanic Niño Index (ONI) es igual o mayor que $+0,5$ ${ }^{\circ} \mathrm{C}$, La Niña cuando dicho valor es igual o menor a $-0,5^{\circ} \mathrm{C}$. Entre ambos valores $(+0,5$ y $-0,5)$ la condición es normal o neutra. Para la reconstrucción diaria de TSM se siguió la metodología descrita en Huang et al. (2015).
El Niño analizado comenzó en noviembre 2014 (ONI: $+0,52$ ) y terminó en mayo 2016 (ONI: +1,08) (NOAA 2017, 2019); aunque los registros de la especie continúan hasta febrero de 2019.

Los registros de la Tabla 1, BM I, BM II, BC, CM y SM fueron realizados por Fundación Ictiológica, con cooperación del Instituto de Fomento Pesquero (IFOP), V. Moncada, M. Montecinos, R. Freire, M. Hüne (Fund. Ictiológica) y E. Reyes (Facultad Ciencias Veterinarias UACh), asociados a la pesquería artesanal de T. atun. Los registros $\mathrm{AU}$ y $\mathrm{CH}$ fueron realizados por el Dr. Patricio Rondini mediante pesca recreativa con moscas de

Tabla 2. Índice oceánico ENOS (ONI) zona Niño 3.4, de 2014 a 2017. Promedio de tres meses para la región $5^{\circ} \mathrm{N}-5^{\circ} \mathrm{S}$ y $120^{\circ} \mathrm{O}-170^{\circ} \mathrm{O} /$ Oceanic Niño Index (ONI) in the Niño 3.4 region, from 2014 to 2017 . Mean of three months for region $5^{\circ} \mathrm{N}-5^{\circ} \mathrm{S}$ and $120^{\circ} \mathrm{W}-170^{\circ} \mathrm{W}$

\begin{tabular}{|c|c|c|c|c|c|c|c|c|c|c|c|c|}
\hline \multirow{3}{*}{$\begin{array}{l}\text { Trimestre } \\
\text { mes }\end{array}$} & \multicolumn{12}{|c|}{$\begin{array}{l}\text { El promedio de tres meses para la región, se considera condición de El Niño cuando ONI es igual o mayor que } \\
0,5^{\circ} \mathrm{C} \text { y La Niña cuando es igual o menor a }-0,5^{\circ} \mathrm{C} \text {. Entre ambos valores el ONI es neutro. }\end{array}$} \\
\hline & DEF & EFM & FMA & MAM & AMJ & MJJ & JJA & JAS & ASO & SON & OND & $\mathrm{NDE}$ \\
\hline & ENE & FEB & MAR & $\mathrm{ABR}$ & MAY & JUN & JUL & AGO & SEP & OCT & NOV & DIC \\
\hline 2017 & $-0,30$ & $-0,10$ & $+0,10$ & $+0,3$ & $+0,4$ & $+0,4$ & $+0,2$ & $-0,1$ & $-0,4$ & $-0,7$ & $-0,9$ & $-1,0$ \\
\hline 2016 & $+2,5$ & $+2,2$ & $+1,7$ & $+1,0$ & $+0,5$ & $+0,0$ & $-0,3$ & $-0,6$ & $-0,7$ & $-0,7$ & $-0,7$ & $-0,6$ \\
\hline 2015 & $+0,6$ & $+0,6$ & $+0,6$ & $+0,8$ & $+1,0$ & $+1,2$ & $+1,5$ & $+1,8$ & $+2,1$ & $+2,4$ & $+2,5$ & $+2,6$ \\
\hline 2014 & $-0,4$ & $-0,4$ & $-0,2$ & $+0,1$ & $+0,3$ & $+0,2$ & $+0,1$ & 0,0 & $+0,2$ & $+0,4$ & $+0,6$ & $+0,7$ \\
\hline
\end{tabular}

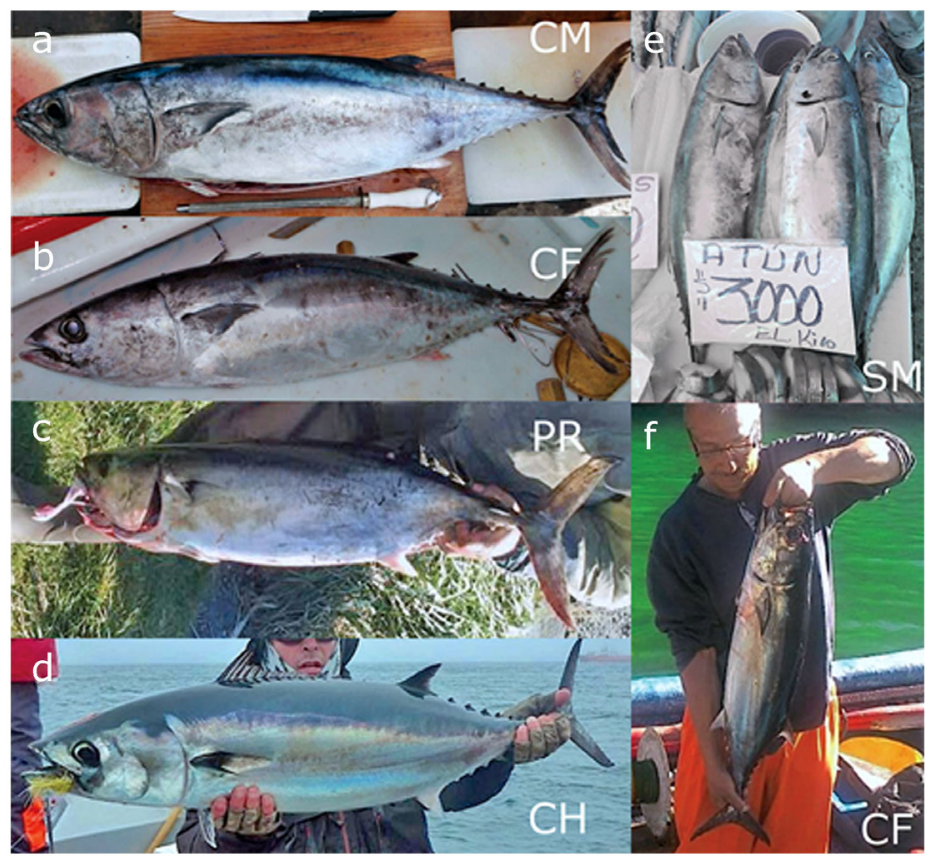

Figura 3. A. fallai estudiados en el sur de Chile: a) CM, Caleta Mehuín 2016; b) CF, Canal Frödden 2017; c) PR, Puerto Riquelme 2017; d) CH, Canal de Chacao 2017 (capturado como pesca recreativa); e) SM, Isla Santa María 2017; f) CF, Canal Frödden 2017 / Availability of A. fallai in southern Chile: a) CM, Caleta Mehuín 2016; b) CF, Canal Frödden 2017; c) PR, Puerto Riquelme 2017; d) CH, Canal de Chacao 2017 (captured as recreational fishing); e) SM, Isla Santa María 2017; and f) CF, Canal Frödden 2017 
Oncorhynchus tshawytscha Walbaum, 1792 (Fig. 3) y P. Reyes (Fund. Ictiológica). El registro PN varó en la costa del Canal Señoret donde fue detectado por Patricio A. Barría de la Agrupación Ecológica Patagónica de Magallanes. El registro PR fue realizado por M. Hüne (Fund. Ictiológica) durante monitoreos de la pesquería artesanal de Merluccius australis (Hutton, 1872), al igual que el registro CF, realizado por M. Martínez de la Corporación de Fomento de la Producción (CORFO) de Aysén y Marco Coronado S. de la flota artesanal extractora de M. australis de Puerto Gala, Canal Moradela (Tabla 1, Figs. 2 y 3 ).

Los recientes registros de la especie al sur de $18^{\circ} \mathrm{S}$ en la costa del Pacífico Suroriental superan cualquier observación histórica de abundancia por unidad de tiempo (Zama \& Cárdenas 1984, Pequeño 1989). Por ello la ocurrencia de decenas de registros, que sustentan incluso su venta en el mercado de Angelmó, Los Lagos (El Llanquihue 2017) ${ }^{1}$ y en el Mercado Fluvial de Valdivia, Los Ríos (Tabla 1) o en el Terminal Pesquero Artesanal La Poza de Talcahuano, región del Biobío (Fig. 3, SM). A partir de 2014 hasta la fecha de entrega de esta investigación (junio 2017), entre las localidades extremas del estudio se seleccionó los 10 registros analizados, en base a la confiabilidad de la identificación y experiencia del muestreador. Los 10 registros analizados superan el único registro previo de dos especímenes en Aysén durante El Niño 1982-83. Los registros actuales, a diferencia de los del 2012 en Aysén, continuaron en presencia de La Niña (agosto 2016) y también con valores neutros o "normales" de ENOS (junio 2016) (Fig. 4).

La identidad de la especie se determinó merística y morfométricamente, mediante Collette \& Nauen (1983). Los resultados de las mediciones morfométricas y merísticas se presentan en las Tablas 3 y 4, donde se comparan con datos de la literatura (Olsen 1962, Roberts 1975, Collette \& Nauen 1983, Zama \& Cárdenas 1984, Collette \& Díaz de Astarloa 2008 y Amorin et al. 2011). Tras la identificación, dos especímenes colectados en BC fueron donados al Museo Nacional de Historia Natural de Chile (MNHNCL), donde se encuentran conservados con los números de colección MNHNCL ICT 7536-1 y MNHNCL ICT 7536-2, correspondiendo éstos a los primeros y únicos ejemplares de la especie preservados en una colección zoológica chilena.

Figura 4. Imágenes satelitales de las Anomalías de la TSM: A) ENOS (BM, mayo a junio 2015); B) Normal (BC, junio 2016), y C) La Niña (CM, agosto 2016) / Satellite images of SSTs: A) El Niño (BM, May to June 2015); B) Normal (BC, June 2016), and C) La Niña (CM, August 2016)
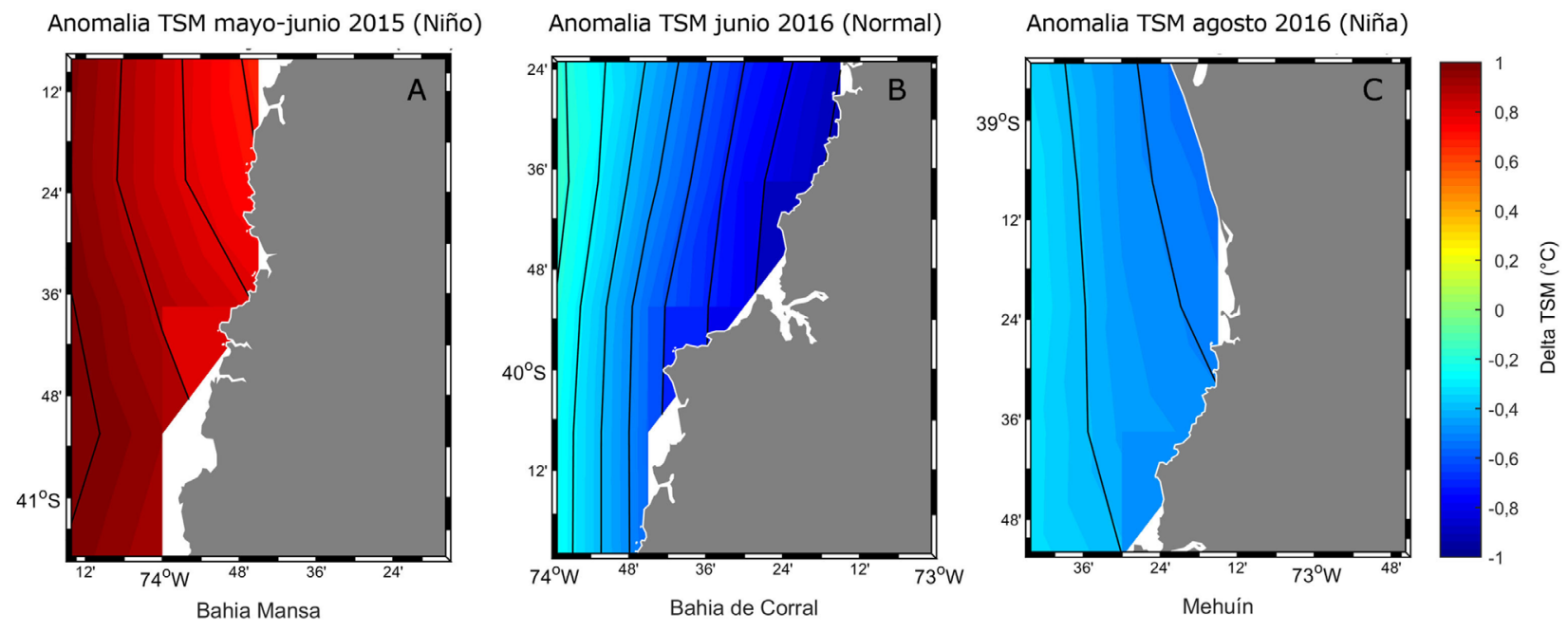
Tabla 3. Morfometría de los especímenes MNHNCL ICT 7536-1 y 2, comparada con datos de la literatura. Medidas expresadas como \% de longitud de horquilla (LH) / Morphometry of specimens MNHNCL ICT 7536-1 and 2, compared with literature data. Measurements are expressed as \% of fork length (LH)

\begin{tabular}{|c|c|c|c|c|c|c|c|c|}
\hline $\begin{array}{c}\text { Ref.:Medida } \\
\quad(\% \text { LH) }\end{array}$ & $\begin{array}{l}\text { MNHNCL } \\
\text { ICT 7536-1 }\end{array}$ & $\begin{array}{l}\text { MNHNCL } \\
\text { ICT 7536-2 }\end{array}$ & $\begin{array}{c}\text { Zama \& } \\
\text { Cárdenas } 1984 \\
(\min -\max n=2)\end{array}$ & $\begin{array}{l}\text { Amorim } \\
\text { et al. } \\
2011\end{array}$ & $\begin{array}{c}\text { Collette \& } \\
\text { Díaz de } \\
\text { Astarloa } \\
2008 \\
\end{array}$ & $\begin{array}{l}\text { Collette } \\
\& \text { Nauen } \\
1983\end{array}$ & $\begin{array}{c}\text { Olsen } 1962 \\
(\min -\max n=3)\end{array}$ & $\begin{array}{c}\text { Roberts } 1975 \\
\text { juv. } \\
(\min -\max n=2)\end{array}$ \\
\hline & $\mathrm{CL}$ & CL & $\mathrm{CL}^{*}$ & BR & AR & $\mathrm{BI}$ & $\mathrm{AU}$ & $\mathrm{AU}$ \\
\hline LH (mm) & 1.020 & 750 & $660-725$ & 685 & 784 & 960 & $760-965$ & $278-282$ \\
\hline L. cefálica & 22,5 & 23,3 & $27,7-28,7$ & 25,7 & 26,0 & - & $20,5-23,0$ & $26,1-26,9$ \\
\hline Pre-D I \% & 28,4 & 28,0 & $31,2-32,3$ & 31,4 & 30,0 & - & 24,2 & $31,3-32,2$ \\
\hline Pre-D II & 53,9 & 56,0 & - & 61,9 & 63,0 & $61-65,4$ & 49,2 & $62,8-63,7$ \\
\hline Pre-V & 26,5 & 20,0 & - & 28,8 & 28,4 & - & 22,0 & $29,5-30,4$ \\
\hline Base D I & 23,5 & 21,5 & - & 29,9 & 31,5 & - & - & $32,3-32,7$ \\
\hline Pre-P & 25,5 & 25,3 & - & 28,8 & 28,4 & - & 21,5 & - \\
\hline Base D II & 6,0 & 6,0 & - & 7,0 & 7,5 & - & - & $5,6-6,4$ \\
\hline Base A \% & 6,0 & 5,0 & $68,3-68,6$ & 7,0 & 7,0 & - & - & $6,1-6,5$ \\
\hline Long. P \% & 11,5 & 10 & $13,9-14,2$ & 13,0 & 14,4 & - & - & - \\
\hline Long. A \% & 7,5 & 6,0 & - & 8,6 & 8,5 & - & - & - \\
\hline D. ojo & 3,2 & 4,4 & 4,7 & 3,6 & 4,1 & - & 3,0 & 3,3 \\
\hline L. Preoral & 7,5 & 6,0 & - & 8,5 & 9,9 & - & 7,0 & 8,0 \\
\hline Dnbo & 9,3 & 10,0 & - & 11,4 & 11,2 & - & - & - \\
\hline
\end{tabular}

Dnbo: Distancia desde la nariz al borde posterior del ojo; juv: Juvenil

*Zama \& Cárdenas (1984) presentan la morfometría en base a longitud estándar (LE)

Tabla 4. Merística de los especímenes MNHN ICT 7536 - 1 y 2, comparada con la literatura / Meristic of MNHNCL ICT $7536-1$ and 2 specimens, compared with literature data

\begin{tabular}{|c|c|c|c|c|c|c|c|c|c|c|}
\hline Ref.: \% LH & $\begin{array}{l}\text { MNHN CL } \\
\text { ICT 7536- } 1\end{array}$ & $\begin{array}{l}\text { MNHN CL } \\
\text { ICT 7536-2 }\end{array}$ & $\begin{array}{c}\text { Zama \& } \\
\text { Cárdenas } \\
1984 \\
\end{array}$ & $\begin{array}{l}\text { Amorim } \\
\text { et al. } \\
2011 \\
\end{array}$ & $\begin{array}{c}\text { Collette \& } \\
\text { Díaz de } \\
\text { Astarloa 2008 }\end{array}$ & $\begin{array}{c}\text { Collette \& Nauen } \\
1983\end{array}$ & $\begin{array}{l}\text { Olsen } 1962 \\
\text { Jul- } 1960\end{array}$ & $\begin{array}{l}\text { Olsen } 1962 \\
\text { ago-61 }\end{array}$ & $\begin{array}{c}\text { Roberts } 1975 \\
\text { juv. } 1\end{array}$ & $\begin{array}{c}5 \text { Roberts } 1975 \\
\text { juv. } 2\end{array}$ \\
\hline & CL & CL & CL & $\mathrm{BR}$ & $\mathrm{AR}$ & BI & $\mathrm{AU}$ & $\mathrm{AU}$ & NZ & NZ \\
\hline Dorsal & $X V-10$ & XVI-10 & $\mathrm{XV}-12$ & XVI-12 & XVII-13 & XV/XVIII-12/13 & $\mathrm{XV}-12$ & XV & $X V-12$ & $\mathrm{XV}-*$ \\
\hline Pínulas D & 7 & 7 & 7 & 7 & 7 & 6 a 7 & 7 & 7 & 7 & 7 \\
\hline Anal & XI-6 & XI-6 & 14 & XIII-7 & XIII-7 & 13 a 14 & XIV-7 & 7 & XIII-7 & XIII-7 \\
\hline Pínulas A & 7 & 7 & 7 & 7 & 7 & 6 a 7 & & - & - & - \\
\hline Pectoral & 26 & 26 & 26 & 27 & 25 & 24 a 26 & 25 & - & 25 & 25 \\
\hline $\mathrm{BE}$ & 75 & 75 & 76 & 73 & 74 & $70-80$ & $76-77$ & 77 & - & 75 \\
\hline PA $\%$ & 65,5 & 47,5 & $68,3-68,6$ & - & - & - & - & - & - & - \\
\hline
\end{tabular}

BE: Branquiespinas, PA\%: Pre-anal, juv: Juvenil

Finalmente, para el análisis se usó antecedentes de la especie contenidos en un Informe Fondo de Fomento para la Pesca Artesanal (FFPA) (Reyes et al. 2017), donde se documenta y oficializa que $A$. fallai correspondió en 20162017 al 0,1\% de la fauna acompañante de la pesca de $T$. atun en la región de Los Ríos, los que despertaron el interés local de la especie como nuevo recurso pesquero artesanal, disponible a público (Fig. 2).
En el presente documento se emplean los siguientes acrónimos: Fondo de Fomento para la Pesca Artesanal (FFPA); Centro Nacional de Información Ambiental (NCEI); Administración Nacional Oceánica y Atmosférica de Estados Unidos de Norteamérica (NOAA); Radiómetro de muy Alta Resolución (AVHRR); FIPA (Fondo de Investigación Pesquera y de Acuicultura); SUBPESCA (Subsecretaría de Pesca y Acuicultura); FFPA (Fondo de Fomento para la Pesca Artesanal); SERNAPESCA (Servicio Nacional de Pesca y Acuicultura); FIC (Fondo de Innovación para la Competitividad); FIC-R (FIC-Regional); CL (Chile); BI (Bibliográfico); BR (Brasil); AR (Argentina); AU (Australia) y; NZ (Nueva Zelanda). 


\section{Resultados}

El estudio morfométrico y merístico de los especímenes donados al MNHNCL (Tablas 3 y 4), permitió identificar la especie como A. fallai, gracias a las siguientes características diagnósticas específicas; relación longitud cabeza/longitud horquilla; morfología y morfometría de las aletas pectorales y, número de pínulas dorsales y ventrales, ente otras (Tablas 3 y 4), incluida la coloración general, presentada en las Figs. 2 y 3.

En noviembre de 2014 ocurre el registro de atún lanzón en CL (PN), junto al primer mes en presentar una condición débil de El Niño (ONI: +0,52) en la región Niño 3.4 del Pacífico Suroriental. Sin embargo, en aguas oceánicas de Magallanes no se presentaron dichos valores, ocurriendo los dos registros de atunes en Magallanes (PN y PR) al menos $200 \mathrm{mn}$ al interior de canales patagónicos, donde la salinidad superficial oscila entre 13,4 y 20 , aumentando hacia la zona oceánica exterior o aguas profundas, bajo los $20 \mathrm{~m}$ de profundidad, donde la salinidad alcanza entre 33 y 33,5 (Silva \& Calvete 2002, Hüne \& Vega 2015). En PN y PR, los valores máximos de TSM durante noviembre de 2014 fueron de $8,6^{\circ} \mathrm{C}$, con su promedio en los primeros $10 \mathrm{~m}$, un $0,76^{\circ} \mathrm{C}$ más bajo que el promedio térmico, que generó un fuerte gradiente vertical. Al contrario, el registro
BM II la columna de agua presentó valores ENOS +0,59 y estratificación térmica, pero no salina (32,8-34,9) (Fig. 5). El oxígeno disuelto en la superficie fue $8,38 \mathrm{mg} \mathrm{L}^{-1}$, la TSM y $\triangle$ TSM fueron $13,8^{\circ} \mathrm{C} \mathrm{y} 0,8^{\circ} \mathrm{C}$, respectivamente (Tabla 1).

Todos los registros ocurridos en aguas chilenas entre noviembre 2014 y mayo 2016 sucedieron durante El Niño (PN, BM, PR, AU, BM II) (Tabla 1). Luego, las capturas de $A$. fallai en condiciones "normales" (ONI: $+0,5$ y $-0,5)$ ocurrieron entre junio y julio de 2016 y luego entre enero y septiembre de 2017, con capturas en $\mathrm{CH}, \mathrm{SM}, \mathrm{CF}$ y BC (Tablas 1 y 2). Finalmente, ocurrieron registros bajo condición La Niña entre agosto y diciembre de 2016, y entre octubre y diciembre de 2017 (CM) (Tabla 1). Los continuos registros de la especie durante El Niño, La Niña y bajo condiciones normales, permiten descartar una conexión vinculante entre la presencia de la especie y El Niño, especialmente en los registros que se realizaron al sur de los $41^{\circ} \mathrm{S}$ (e.g., PN, PR, AU, CF \& CH) (Tabla 1 y 2), donde El Niño no se manifiesta en la TSM, salvo que la intensidad del fenómeno sea grande (Zama \& Cárdenas 1984, Lehodey 2001, Carr et. al. 2002, Rocha 2007, Grendi et. al. 20173). Por lo tanto, los resultados indican que una $\Delta$ TSM positiva o cálida debido a un evento de El Niño no es indicador directo de la presencia de $A$. fallai en los lugares costeros de registro (Tabla 1).
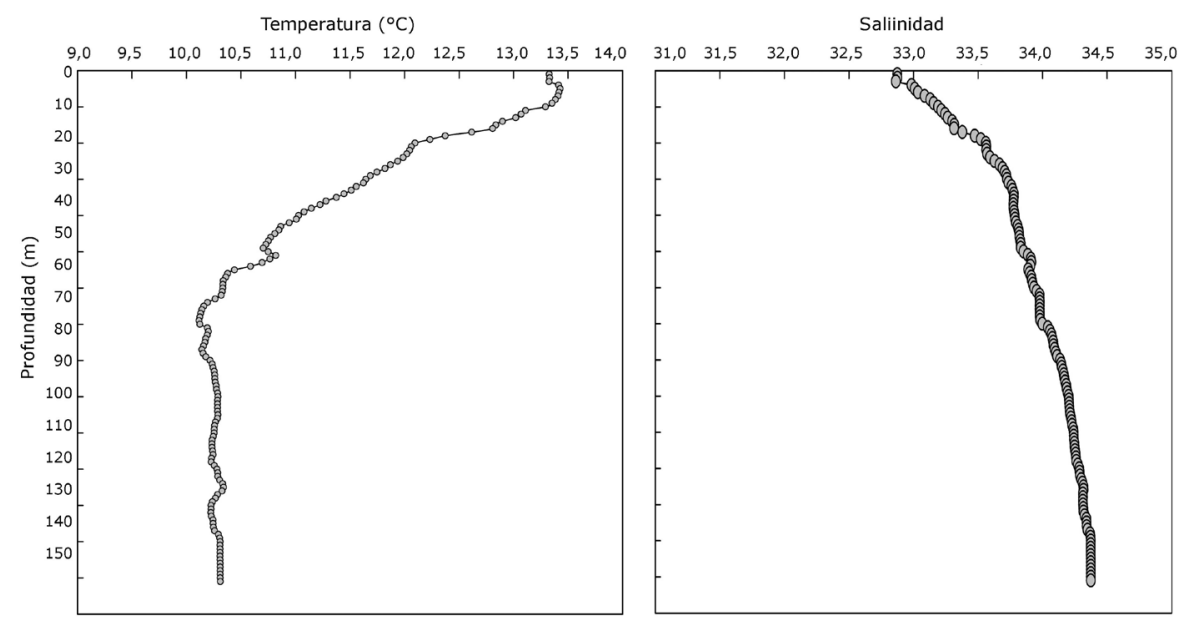

Figura 5. Perfil de temperatura y salinidad de la columna de agua en BM II el 15 de mayo 2016 / Temperature and salinity profile of BM II on May 15, 2016

\footnotetext{
${ }^{3}$ Grendi C, H Reyes, M Pizarro, U Cifuentes \& A Bustamante. 2017. Efectos de El Niño 2015-16 sobre las condiciones oceanográficas en la costa de Chile entre $18^{\circ}$ y $41^{\circ} \mathrm{S}$. Instituto de Fomento Pesquero (IFOP), Valparaíso. Libro de Resúmenes, XXXVII Congreso Sociedad Chilena de Ciencias del Mar, Valparaíso, p. 494.
} 
Respecto a sus registros al interior de los canales de la región de Magallanes y Aysén (PN, PR y CF, Fig. 1), allí la haloclina suele hallarse a $20 \mathrm{~m}$ de profundidad, donde la salinidad alcanza 33, encontrándose esos valores en el rango de habitabilidad de los peces oceánicos, como los atunes (Bustos et. al. 2008, Pepperell \& Harvey 2010), lo cual facilita su acceso a través del sistema de canales patagónicos, localizados a más de $100 \mathrm{mn}$ de la costa oceánica.

Los resultados del presente estudio se enfocan en el sur de Chile ( $\left.37^{\circ} 03^{\prime}-51^{\circ} 49^{\prime} \mathrm{S}\right)$. Sin embargo, los registros y su permanente presencia en mercados de peces y actividades de pesca recreativa se observó en todo el país (18² $28^{\prime} \mathrm{S}-$ $51^{\circ} 49^{\prime}$ S), lo que motivó a SUBPESCA a oficializar su condición de Nuevo Recurso Pesquero el 5 de junio de 2017 (SUBPESCA 2017) ${ }^{4}$, incorporándolo como fauna acompañante de $X$. gladius con pesca de "palangre" y $M$. australis con "espinel" entre los $18^{\circ} 28^{\prime} \mathrm{S}$ y $41^{\circ} 28^{\prime} \mathrm{S}$. En 2018 SUBPESCA lo incorporó como fauna acompañante de T. atun con "línea de mano" en Los Ríos (3948'S), donde en 2016-2017 la especie representó el 0,1\% de la fauna acompañante de $T$. atun, igualando en volumen el desembarque de T. murphyi (Reyes et al. 2017, SUBPESCA $\left.2018^{5}\right)$.

\section{Discusión}

Inicialmente se planteó como hipótesis que los registros de atún lanzón en aguas costeras del sur de Chile se debían a incrementos costeros de la TSM asociados a El Niño 20142016, ello con el único antecedente previo en Chile, ocurrido durante un evento de El Niño muy intenso, denominado "meganiño 1982-83" o "la madre de todos los niños" (1982-1983) (Zama \& Cardenas 1984, Maturana et al. 1997, Rocha 2007, Grendi et. al. 2017), y además, la hipótesis se sustentaba porque todos los registros desde 2014 hasta mayo de 2016 ocurrieron durante El Niño (Tablas 1 y 2).

No obstante lo anterior, a partir de junio de 2016, bajo condiciones "normales" o bajo condición La Niña, continuaron los registros de $A$. fallai, que desacreditan la hipótesis inicial (Tablas 1 y 2) y generan la búsqueda de una nueva hipótesis alternativa, que indica que el arribo de la especie en aguas costeras del sur de Chile se generaría por efecto de El Niño en aguas oceánicas, donde genera efectos negativos en la abundancia de zooplancton (Yatsu 1995, González et al. 1998). Durante un evento de El Niño se genera en el ecosistema oceánico un menor suministro de nutrientes, un incremento de la temperatura y al desplazamiento de las isotermas hacia aguas costeras (Carr et. al. 2002), generando cambios en la distribución, biomasa y movimientos en túnidos (Lehodey 2001). El acercamiento geográfico del $A$. fallai a la costa se facilita por el desplazamiento de las isotermas y ventanas térmicas costeras generadas durante un evento de El Niño en la corriente de Humboldt (González et. al. 1998), permitiéndoles acercarse al continente en busca de alimento (Carr et al. 2002, Lehodey 2001, Strub et al. 1998). De esta forma, pueden permanecer cerca de la costa gracias a su capacidad de retener calor y mantener sus cuerpos más calientes que la temperatura circundante (Graham \& Dickson 2000, Sepúlveda et al. 2007, 2008). Por otra parte, su presencia como fauna acompañante de la pesquería de $T$. atun en BC Chile (Tabla 1), deja en evidencia que tal como ocurre en aguas costeras de Nueva Zelanda (O'Driscoll \& McClatchie 1998), la especie cuenta con registros junto a $T$. murphyi forrajeando Euphausida, Cephalopoda y pequeños pelágicos (sardinas y anchoas) (Yatsu 1995, Bertrand et al. 2004, Duarte et al. 2007, Reyes et al. 2017).

Los resultados del presente estudio se enfocan en el sur de Chile ( $\left.37^{\circ} 03^{\prime}-51^{\circ} 49^{\prime} \mathrm{S}\right)$. Sin embargo, el Estado Chileno en 2017 y 2018 incorporó al atún lanzón en la nómina de especies asociadas a pesquerías, entre los $18^{\circ} 28^{\prime} \mathrm{S}$ y $41^{\circ} 28^{\prime} \mathrm{S}$ (SUBPESCA 2017 $\left.7^{4}, 2018^{5}\right)$.

\section{Agradecimientos}

A Daniela Cajas R. de la Dirección Zonal de Pesca y Acuicultura de las regiones de La Araucanía y Los Ríos (SUBPESCA), a Richard Navarro G. del Fondo de Fomento para la Pesca Artesanal y a Germán Pequeño R. del Servicio Nacional de Pesca y Acuicultura de Los Ríos (SERNAPESCA), por sus comentarios. A Fundación Ictiológica, por su patrocinio. A dos evaluadores anónimos por sus contribuciones. A don Marco Ide de la Federación Interregional de Pescadores Artesanales del Sur-Valdivia (FIPASUR) por su apoyo logístico.

\footnotetext{
${ }^{4}$ SUBPESCA. 2017. Resolución Exenta № 1838-17 de la Subsecretaría de Pesca y Acuicultura que Modifica Resolución Exenta № 3115 de 2013 , de esta Subsecretaría de Pesca y Acuicultura. Valparaíso, 5 de junio 2017. Ministerio de Economía, Fomento y Turismo, Subsecretaría de Pesca y Acuicultura, Memo (R.PESQ.) №120 2017. Modifica Nómina Nacional de Pesquerías Artesanales
}

\footnotetext{
${ }^{5}$ SUBPESCA. 2018. Resolución Exenta № 2731-18 de la Subsecretaría de Pesca y Acuicultura que Modifica Resolución Exenta № 3115 de 2013 , de esta Subsecretaría de Pesca y Acuicultura. Valparaíso, 1 de agosto 2018. Ministerio de Economía, Fomento y Turismo, Subsecretaría de Pesca y Acuicultura, Memo (R.PESQ.) №200 2017. Modifica Nómina Nacional de Pesquerías Artesanales
} 


\section{LITERATURA CITADA}

Arana P, S Palma, N Silva, A De Caso \& R Rolliri. 2012. Recursos pesqueros del mar de Chile, 308 pp. Escuela de Ciencias del Mar, Pontificia Universidad de Valparaíso, Valparaíso.

Amorim A, A Largacha \& M Gonzalez. 2011. Occurrence of Slender Tuna (Allothunnus fallai) off Sao Paulo State, Brazil. Collective Volume of Scientific Papers ICCAT 66(5): 2134-2139.

Bahamonde N \& G Pequeño. 1975. Peces de Chile. Lista sistemática. Museo Nacional de Historia Natural de Chile, Publicación Ocasional 21: 1-20.

Blanco JL, ME Carr, AC Thomas \& PT Strub. 2002. Hydrographic conditions off northern Chile during the 1996-1998 La Nina and El Nino events. Journal of Geophysical Research, Oceans 107(C3): 1-19. $<$ doi:10.1029/2001JC001002>

Bustos C, M Landaeta \& F Balbontín. 2008. Efectos ambientales sobre la variabilidad espacial del ictioplancton de Chile austral durante noviembre de 2005. Revista Chilena de Historia Natural 81(2): 205-219.

Carr ME, PT Strub, AC Thomas \& JL Blanco. 2002. Evolution of 1996-1999 La Niña and El Niño conditions off the western coast of South America: A remote sensing perspective. Journal of Geophysical Research, Oceans 107(C12), 3236. <doi:10.1029/2001JC001183>

Collette B \& J Díaz de Astarloa. 2008. Southernmost occurrence of the slender tuna, Allothunnus fallai, off Tierra del Fuego, South Atlantic Ocean. Ichthyological Research 55(1): 95-96.

Collette BB \& CE Nauen. 1983. FAO Species Catalogue. Vol. 2. Scombrids of the world. An annotated and illustrated catalogue of tunas, mackerels, bonitos and related species known to date. FAO Fisheries Synopsis 125(2): 1-137.

Duarte F, C Ibáñez \& J Chong. 2007. Cambios en la morfometría bucal y su relación con la dieta de Thyrsites atun (Euphrasen, 1791) en el centro-sur de Chile. Revista Chilena de Historia Natural 80(4): 407-417.

Eschmeyer W, E Herald \& H Hammann. 1983. A field guide to Pacific coast fishes of North America, 336 pp. Houghton Mifflin Company, Boston.

FAO. 2018. FAO Capture Production of Allothunnus fallai (t). Fishery Statistical Collections Global Capture Production. Fisheries and Aquaculture Department, United Nations (UN). Food and Agriculture Organization, Rome. <http:// www.fao.org/fishery/statistics/global-capture-production/ en>

Fowler H. 1945. Fishes of Chile. Systematic catalog. Apartado de la Revista Chilena de Historia Natural, Part I and II: 1-171.

González H, G Daneri, D Figueroa, JL Iriarte, N Lefevre, G Pizarro, R Quiñones, M Sobarzo \& A Troncoso. 1998. Producción primaria y su destino en la trama trófica pelágica y océano profundo e intercambio océano-atmósfera de $\mathrm{CO}_{2}$ en la zona norte de la Corriente de Humboldt $\left(23^{\circ} \mathrm{S}\right)$ : posibles efectos del evento El Niño, 1997-1998 en Chile. Revista Chilena de Historia Natural 71(4): 429-458.
Graham J \& K Dickson. 2000. The evolution of thunniform locomotion and heat conservation in scombrid fishes: new insights based on the morphology of Allothunnus fallai. Zoological Journal of the Linnean Society 129(4): 419-466.

Huang B, VF Banzon, E Freeman, J Lawrimore, W Liu, TC Peterson, TM Smith, PW Thorne, SD Woodruff \& HM Zhang. 2015. Extended reconstructed sea surface temperature version 4 (ERSST. v4). Part I: Upgrades and intercomparisons. Journal of Climate 28(3): 911-930.

Hüne M \& R Vega. 2015. Spatial variation in the diet of Patagonotothen tessellata (Pisces, Nototheniidae) from the fjords and channels of southern Chilean Patagonia. Polar Biology 38: 1613-1622.

Ichikawa W \& T Shirasawa. 1980. Report on 1979 tuna longline fishing investigation in higher latitudes of eastern South Pacific. Japan Marine Fishery Resources Research Center. Report 23: 1-147.

Lehodey P. 2001. The pelagic ecosystem of the tropical Pacific Ocean: dynamic spatial modelling and biological consequences of ENSO. Progress in Oceanography 49(14): 439-468.

Maturana J, M Bello \& M Manley. 1997. Antecedentes históricos y descripción del fenómeno El Niño, Oscilación del Sur. En: Avaria S, J Carrasco, J Rutllant \& E Yáñez (eds). El Niño-La Niña 1997-2000. Sus efectos en Chile, pp. 13-27. CONA, Valparaíso. <http://www.divulgameteo.es/uploads/ ENSO-antecedentes-descripci\%C3\%B3n.pdf>

Meléndez R, O Gálvez \& A Cornejo. 1993. Catálogo de la colección de peces depositada en el Museo Nacional de Historia Natural de Chile. Publicación Ocasional, Museo Nacional de Historia Natural, Chile 47: 1-233.

Mori K. 1967. Record of occurrence and some notes on Young slender tuna (Allothunnus fallai Serventry) from the stomachs of longline tunas and marlins in the South Pacific Ocean. Report of Nankai Regional Fisheries Research Laboratory, Japan 25: 113-120.

NOAA. 2017. ENSO: Recent Evolution, Current Status and Predictions. Climate Prediction Center (NCEP) 1 May 2017, 32 pp. <http://www.cpc.ncep.noaa.gov/products/analysis monitoring/lanina/enso_evolution-status-fcsts-web.pdf>

NOAA. 2019. ENSO: Recent Evolution, Current Status and Predictions. Climate Prediction Center (NCEP) 25 February 2019, 32 pp. <https://www.cpc.ncep.noaa.gov/products/ analysis_monitoring/lanina/enso_evolution-status-fcstsweb.pdf $>$

O'Driscoll R \& S McClatchie. 1998. Spatial distribution of planktivorous fish schools in relation to krill abundance and local hydrography off Otago, New Zealand. Deep Sea Research, Part 2 45(7): 1295-1325.

Olsen A. 1962. Allothunnus fallai Serventy: A new record for Australian waters. Papers and Proceedings of the Royal Society of Tasmania 96: 1-95.

Pepperell J \& G Harvey. 2010. Fishes of the Open Ocean, 260 pp. University of Chicago Press, Chicago.

Pequeño G. 1989. Peces de Chile. Lista revisada y comentada. Revista de Biología Marina 24(2): 1-132. 
Pequeño G. 1997. Peces de Chile. Lista sistemática revisada y comentada: Addendum. Revista de Biología Marina 32(2): 77-94.

Reyes P \& M Hüne. 2012. Peces del sur de Chile, 500 pp. Editorial Ocho Libros, Santiago de Chile.

Reyes P, M Hüne, P Carimán, A Hernández, P Oyarzún \& M Montecinos. 2017. Asistencia técnica para la implementación de un estudio biológico- pesquero de la sierra Thyrsites atun en la región de Los Ríos. Proyecto Fondo de Fomento para la Pesca Artesanal. Informe Final. Fundación Ictiológica, 01/2017: 1-207. <https://www. fundacionictiologica.org/publicaciones $>$

Roberts P. 1975. Juvenile slender tuna from New Zealand. New Zealand Journal of Marine and Freshwater Research 9(1): 105-108.

Rocha F. 2007. El Meganiño 1982-83, "la madre de todos los niños", y la rehabilitación de las zonas afectadas. Conferencia II Congreso Internacional "Obras de Saneamiento, Hidráulica, Hidrología y Medio Ambiente" HIDRO 2007 ICG. Lima, 06/2007: 1-41.<http://www.imefen.uni.edu.pe/ Temas_interes/ROCHA/FEN_1982-1983.pdf $>$

Sepúlveda C, K. Dickson, L Frank \& J Graham. 2007. Cranial endothermy and a putative brain heater in the most basal tuna species, Allothunnus fallai. Journal of Fish Biology 70(6): 1720-1733.

Sepúlveda C, K Dickson, D Bernal \& J Graham. 2008. Elevated red myotomal muscle temperatures in the most basal tuna species, Allothunnus fallai. Journal of Fish Biology 73(1): 241-249.

SERNAPESCA. 2012. Anuario estadístico de pesca. Serie 2002-2012. Servicio Nacional de Pesca, Gobierno de Chile, Valparaíso. <www.sernapesca.cl/sites/default/files/2012_ series_2002_2012_1.xls>
SERNAPESCA. 2016. Anuario estadístico de pesca 2016. Servicio Nacional de Pesca, Gobierno de Chile, Valparaíso. $<$ http://www.sernapesca.cl/index.php?option=com remository\&Itemid $=246 \&$ func $=$ select\&id $=1207>$

Serventy D. 1948. Allothunnus fallai a new genus and species of tuna from New Zealand. Records of the Canterbury Museum 5(3): 131-135.

Silva N \& C Calvete. 2002. Características oceanográficas físicas y químicas de canales australes chilenos entre golfo de Penas y el estrecho de Magallanes (Crucero CIMAR-Fiordo 2). Ciencia y Tecnología del Mar 25(1): 23-88.

Strub P, J Mesias, V Montecino, J Rutllant \& S Salinas. 1998. Coastal ocean circulation off western South America. In: Robinson AR \& KH Brink (eds). The Sea 11: 273-313, John Wiley, New York.

Warashina I \& K Hisada. 1972. Geographical distribution and body length composition of two tuna-like fishes, Gasterochisma melamphus Richardson and Allothunnus fallai Serventry, tasken by Japanese tuna longline fishery. Bulletin of Far Seas Fisheries Research Laboratory, Japan 6: 51-75.

Watanabe H, M Yukinawa, S Nakazawa \& S Ueyanagi. 1966. On the larvae probably referable to slender tuna, Allothunnus fallai, Serventy. Reports of the Nankai Regional Fisheries Research Laboratory 23: 85-93.

Yatsu A. 1995. The role of slender tuna, Allothunnus fallai, in the pelagic ecosystems of the South Pacific Ocean. Japanese Journal of Ichthyology 41(4): 367-377.

Zama A \& E Cardenas. 1984. Descriptive catalogue of marine and freshwater fishes from the Aysén Region, Southern Chile, with zoogeographical notes on the fish fauna, 75 pp. Servicio Nacional de Pesca, Ministerio de Economía Fomento y Reconstrucción / Japan International Cooperation Agency, Santiago/Tokyo. 